\title{
Programação Linear: Uma Contextualização a partir de Sistemas Lineares
}

\author{
Linear Programming: A Contextualization by using Linear Systems
}

\author{
Marcelo Simplício de Lyra ${ }^{* 1}$ e Thiago Alves de Queiroz ${ }^{2}$ \\ 1,2 Universidade Federal de Goiás - Pólo Catalão, GO, Brasil
}

\begin{abstract}
Resumo
Neste artigo é apresentado uma proposta de introdução da programação linear no ensino médio considerando os métodos de ensino, o perfil do professor, o perfil do estudante e a não flexibilização do currículo escolar. Essa proposta veio como resultado de uma pesquisa de pós-graduação nível Stricto Sensu, que trata de problemas simples para incentivar o estudante a conhecer a Programação Linear de forma contextualizada a conteúdos já conhecidos, como resolução de sistemas lineares. O incentivo é mostrar ao estudante a gama de problemas reais que podem ser resolvidos usando Programação Linear, com a aplicação de métodos para lidar com sistemas de equações lineares e o apoio de softwares matemáticos. Apesar da proposta não ter sido aplicada em sala de aula, estima-se que o aluno será capaz de compreender de forma contextualizada onde se aplica sistemas lineares, a importância da modelagem matemática e a necessidade de utilizar um software para resolver problemas de grande porte.
\end{abstract}

Palavras-chave: Ensino médio. Equações lineares. Otimização. Software.

\begin{abstract}
This paper presents a proposal for the introduction of linear programming in high school considering teaching methods, the teacher and student profiles, and a non-flexibilization of the curriculum. This proposal came as a result of a Stricto Sensu research, which deals with simple problems that encourage high school students to understand Linear Programming by means of a contextualization of studied topics, as resolution of linear equations. The aim is to show to these students the range of real-world problems that can be solved using Linear Programming, with the application of methods to solve systems of linear equations and the support of mathematical softwares. The proposal presented here has not been applied in classrooms yet, but it is expected that students will be able to understand in a contextualized view where linear systems can be applied, the importance of mathematical modeling and the need of to use a mathematical software to solve real big data problems.
\end{abstract}

Keywords: High school. Linear equations. Optimization. Software. 


\section{Introdução}

A busca pela melhoria do ensino da Matemática e a adequação do seu currículo nas escolas de ensino médio incentivam cada vez mais professores a criar formas de se trabalhar o conhecimento. Assim, o artigo aqui apresentado busca apresentar uma proposta didática para o ensino da programação linear por meio de uma contextualização do conteúdo sistemas lineares, sendo observado principalmente às metodologias usuais, as diversas tendências de ensino e a questão curricular.

A base para este estudo está na utilização de situações problemas do cotidiano, para os quais se constroem modelos utilizando uma ferramenta matemática simples: sistema de equações lineares. Conforme Leon (2011), grande parte dos problemas matemáticos existentes na ciência ou na indústria envolvem em algum momento a resolução de sistemas lineares. Além disso, problemas de programação linear envolvem muitas variáveis, porém se estudados em um contexto menor, esperase um aprendizado por parte de alunos do ensino médio e a possível extensão para os casos mais complexos. A programação linear é um exemplo que tem permitido lidar com situações problema em diversas áreas do conhecimento, como na economia, administração, engenharia, agronomia e indústria. Os problemas são modelados por meio de equações lineares, resultando em um modelo de otimização.

O tema programação linear atualmente é uma das aplicações mais evidentes no âmbito matemático, principalmente nas questões de sustentabilidade, tema amplamente discutido internacionalmente, mas que infelizmente não é tratado no ensino médio da maioria das escolas de nosso país. Nesse sentido, pode-se dizer de uma forma bem simples que otimizar é essencial para o bom desenvolvimento de uma sociedade, pois o conceito de otimizar é utilizado para a busca de resultados mais eficientes, de forma que: gastar menos recursos, maximizar lucros, minimizar tempo, e etc., são denominações simples que podem fazer parte do dia a dia dos alunos.

Alguns autores já trabalharam com a proposta de metodologias para o ensino médio. Melo (2012), por exemplo, abordou primeiramente o problema de programação linear no aspecto de quantidades de soluções com exemplos reais. Em seguida, o processo de modelagem e aspectos da resolução de problemas foram discutidos por meio de diferentes abordagens. Também, o autor fez um breve histórico do ensino da programação Linear no ensino médio.

Almeida (2013) trabalhou com o teorema fundamental da Programação Linear e sua aplicação no ensino médio. Este autor discutiu sobre a existência de soluções para tais problemas observando a região poliedral do modelo matemático associado.

O trabalho de Silva (2013) aborda a modelagem matemática no contexto do ensino médio, criando uma relação com o uso de recursos computacionais. Na resolução de problemas de programação linear o autor utilizou o método gráfico e o método Simplex com o auxílio de softwares computacionais, como o solver do Microsoft Excel.

Esses autores citados propuseram uma abordagem diferente da que é considerada neste trabalho. Baseia-se na teoria da programação linear, de forma que busca por passos simples para a resolução dos problemas, que não requerem que novos conteúdos sejam abordados, apenas uma extensão dos conceitos previstos no currículo atual. Então, conceitos como o de combinatória, para o cálculo da quantidade total de soluções, e resolução de sistemas de equações lineares com duas ou três variáveis, que são temas abordados no ensino médio, fazem-se necessário. O objetivo dessa proposta é inserir a programação linear no estudo de tópicos já abordados no currículo do ensino médio, a partir de uma perspectiva prática (ZEICHNER, 1993), e por meio da tendência construtivista (FIORENTINI, 1995).

Para tanto este artigo está dividido em seções. $\mathrm{Na}$ Seção 2 apresenta-se uma discussão teórica sobre modelagem matemática e resolução de sistemas de equações lineares, enfatizando a importância da utilização, em sala de aula, de situações do dia a dia do estudante. Na Seção 3, apresenta-se a metodologia proposta para resolver os problemas/modelos de programação linear por meio da resolução de vários sistemas lineares. $\mathrm{Na}$ penúltima, Seção 4, um software matemático é apresentado como ferramenta para lidar com os vários sistemas de equações lineares que surgem no decorrer do processo. Por fim, considerações finais e direções para trabalhos futuros são dadas na Seção 5 .

\section{Modelagem na Programação Linear}

Com o objetivo de introduzir a programação linear no ensino médio, para se resolver situações problemas que envolvam duas ou três variáveis, a 
proposta deste trabalho busca: abordar a modelagem matemática, compreender a situação problema, criar modelos, abordar a resolução de sistemas de equações lineares, obter a solução do modelo.

Ao lidar com problemas de programação linear, busca-se maximizar ou minimizar uma função, chamada de objetivo, conjuntamente com várias restrições impostas ao problema dado, que são caracterizadas por um sistema de equações/inequações lineares. Essas restrições incluem características do problema quanto à limitação de recursos, como por exemplo: mão de obra, quantidade de matéria prima, requisitos financeiros, entre outros.

Segundo Bassanezi (2002), a modelagem matemática pode ser encarada como um método de pesquisa científico e uma estratégia de ensinoaprendizagem. Por isso, o papel do professor é fundamental no contexto da modelagem matemática. Para Polya (1995) é importante que o professor incentive o aluno a se tornar independente quanto à abordagem de qualquer problema. Então, é primordial que o professor dose o auxílio ao seu aluno, uma vez que se o professor auxiliar pouco, o aluno não conseguirá um progresso significativo. Por outro lado, se o professor auxiliar muito não resta parte significativa para o aluno executar. Nesse sentido, é interessante que o professor seja empático em relação ao aluno e principalmente discreto no seu auxílio.

Segundo Biembengut e Hein (2000) e Bassanezi (2002), a obtenção do modelo matemático por meio de um problema real perpassa as seguintes etapas: (i) reconhecimento do problema; (ii) formulação do problema; e, (iii) validação da solução. Na etapa (i) ocorre a interação com o assunto e a busca pelo conhecimento necessário para o estudo da situação problema. Na etapa (ii) ocorre a interpretação para a linguagem matemática e a resolução do problema. É neste momento que se faz a identificação das variáveis de decisão e a representação do problema em simbologia algébrica. Também, faz-se a identificação das restrições e a devida representação por meio de equações ou inequações lineares e a identificação do objetivo do problema. Na etapa (iii) verifica-se quanto o modelo e a respectiva solução se aproximam da situação problema em estudo.

No contexto da programação linear, a solução de um modelo é obtida por meio de algoritmos computacionais, uma vez que boa parte dos problemas envolvem modelos com centenas ou milhares de variáveis e restrições. Um algoritmo bastante utilizado neste caso é o Simplex (ARENALES et al., 2007), porém ensiná-lo no contexto do ensino médio requer tempo $\mathrm{e}$ flexibilização curricular, pois novos conteúdos precisariam ser inseridos. Ambas as necessidades impõe que o cronograma e as metas estabelecidas para o ensino de acordo com a instituição/governo sejam repensadas, o que não é uma tarefa simples, muito menos de curto prazo.

Portanto é interessante utilizar conteúdos e abordagens previstas nos currículos, que é o caso do estudo da modelagem e sistemas de equações lineares. Com isso, o contexto de ensino explora um conjunto de restrições representado por um sistema de inequações lineares, que é, então, transformado em um conjunto de equações lineares. Durante a resolução do modelo, que envolve até três variáveis de decisão, cabe finalmente lidar com um sistema de equações.

Uma abordagem a ser seguida pelo professor consiste em apresentar o problema, caracterizando-o em um contexto simples e objetivo para o aluno. É interessante sempre começar por problemas que envolvam modelos com duas variáveis para, então, abordar problemas com três ou mais variáveis. Deve-se deixar claro que o modelo resultante impacta no resultado final, então muita atenção deve ser tomada no passo de modelagem. Também, enfatizar para os alunos que o primeiro passo a ser seguido, no reconhecimento do problema, consiste em determinar as variáveis de decisão, pois a partir delas é que se determinam as equações/inequações que regem o modelo, isto é, as restrições e a função objetivo. Em outras palavras, os alunos devem escrever o modelo na forma padrão:

$$
\begin{gathered}
\text { Minimizar } \mathrm{z}=c_{1} x_{1}+c_{2} x_{2}+\ldots \ldots \ldots+c_{n} x_{n} \\
\text { Sujeito a: }\left\{\begin{array}{c}
a_{11} x_{1}+a_{12} x_{2}+\ldots \ldots \ldots+a_{1 n} x_{n}=b_{1} \\
a_{21} x_{1}+a_{22} x_{2}+\ldots \ldots \ldots+a_{2 n} x_{n}=b_{2} \\
\cdot \\
\cdot \\
a_{m 1} x_{1}+a_{m 2} x_{2}+\ldots \ldots \ldots+a_{m n} x_{n}=b_{m} \\
x_{2} \geq 0, x_{2} \geq 0, \ldots \ldots, x_{n} \geq 0
\end{array}\right.
\end{gathered}
$$

De acordo com a eq. (1), a função $z$ a ser minimizada, que poderia também ser maximizada, é chamada de função objetivo. O sistema de equações lineares representa as restrições do problema, enquanto a última linha delimita o domínio das variáveis, que neste caso diz que cada uma pode 
no modelo são números reais expressos por $c_{j}, a_{i j} \mathrm{e}$ $b_{i}$ para $i=1,2, \ldots, m$ e $j=1,2, \ldots, n$.

Durante a modelagem pode surgir uma inequação, por exemplo, $x_{1}+x_{2} \leq 0$, que pode ser "completada" até se obter uma igualdade. Em outras palavras, com a introdução de uma variável $y \in \mathbb{R}$, com $y \geq 0$, chega-se na seguinte equação linear, $x_{1}+$ $x_{2}+z=0$, em que $z$ representa a "folga" da inequação para se atingir a igualdade. Se por outro lado, a inequação for $x_{1}+x_{2} \geq 0$, tem-se um "excesso" na igualdade, de forma que uma nova variável $w \in \mathbb{R}$, com $w \geq 0$ entra na restrição com coeficiente negativo, resultando em $x_{1}+x_{2}-w=0$ (HILLIER; LIEBERMAN, 2008).

\subsection{Sistemas Lineares}

Nesse trabalho o interesse está em modelos de programação linear com duas e três variáveis para que seja possível usar os conceitos e o estudo a ser desenvolvido nas aulas de sistemas de equações lineares. O professor pode iniciar o trabalho com os alunos usando sistemas com coeficientes genéricos para mostrar a generalidade dos resultados. Ao obter o valor das variáveis em função dos coeficientes e termos independentes, o aluno tem fórmulas para resolver os sistemas com valores numéricos, apenas fazendo a devida substituição do valor pelo respectivo coeficiente/termo independente genérico. Parte-se do seguinte sistema linear com duas variáveis:

$$
\left\{\begin{array}{l}
a_{11} x_{1}+a_{12} x_{2}=b_{1} \\
a_{21} x_{1}+a_{22} x_{2}=b_{2}
\end{array}\right.
$$

Algumas técnicas que podem ser utilizadas pelos alunos para buscar a solução de sistemas lineares consistem em aplicar o método da substituição ou o método da adição, por exemplo. Outros métodos também podem ser usados, como o método da comparação ou o método da eliminação de GaussJordan. Mais detalhes em Howard e Rorres (2001).

Ao aplicar o método da soma na eq. (2), chega-se no seguinte resultado:

$$
\begin{aligned}
& x_{1}=\frac{a_{22} b_{1}-a_{12} b_{2}}{a_{11} a_{22}-a_{21} a_{12}} \\
& x_{2}=\frac{a_{21} b_{1}-a_{11} b_{2}}{a_{21} a_{12}-a_{11} a_{22}}
\end{aligned}
$$

Para sistemas lineares cujo número de variáveis e equações é maior do que dois, o método da soma, por exemplo, apresenta a dificuldade da intensa exemplos numéricos. Por isso, o professor pode usar o método de eliminação de Gauss-Jordan para sistemas maiores (BOLDRINI et al., 1980).

No caso de sistemas lineares com três variáveis e três equações, ao aplicar o método de Gauss-Jordan, o primeiro passo consiste em escrever a matriz ampliada do sistema linear, isto é:

$\left\{\begin{array}{l}a_{1} x+b_{1} y+c_{1} z=d_{1} \\ a_{2} x+b_{2} y+c_{2} z=d_{2} \\ a_{3} x+b_{3} y+c_{3} z=d_{3}\end{array} \Rightarrow\left(\begin{array}{lll|l}a_{1} & b_{1} & c_{1} & d_{1} \\ a_{2} & b_{2} & c_{2} & d_{2} \\ a_{3} & b_{3} & c_{3} & d_{3}\end{array}\right)\right.$

As operações elementares que podem ser aplicadas na matriz ampliada do sistema linear são: (i) permuta de linhas; (ii) multiplicação (ou divisão) de uma linha por um escalar diferente de zero; e, (iii) adicionar uma linha com uma combinação linear das demais linhas. Estas operações não alteram o conjunto solução do sistema linear, tal que uma demonstração para esta afirmação pode ser obtida em Boldrini et al. (1980).

Aplicando as operações elementares necessárias no eq. (5), desenvolve-se o sistema de forma genérica, conseguindo sistemas equivalentes ao sistema dado, ou seja:

$$
\left(\begin{array}{ccc|c}
1 & \frac{b_{1}}{a_{1}} & \frac{c_{1}}{a_{1}} & \frac{d_{1}}{a_{1}} \\
0 & 1 & \frac{a_{1} c_{2}-a_{2} c_{1}}{a_{1} b_{2}-a_{2} b_{1}} & \frac{a_{1} d_{2}-a_{2} d_{1}}{a_{1} b_{2}-a_{2} b_{1}} \\
0 & 0 & k_{1} & k_{2}
\end{array}\right)
$$

sendo:

$$
\begin{aligned}
& k_{1}=\frac{a_{1}\left(b_{2} c_{3}-b_{3} c_{2}\right)-a_{3}\left(b_{2} c_{1}+b_{1} c_{2}\right)-a_{2}\left(b_{1} c_{3}-b_{3} c_{1}\right)}{a_{1} b_{2}-a_{2} b_{1}} \\
& k_{2}=\frac{a_{1}\left(b_{2} d_{3}-b_{3} d_{2}\right)-a_{3}\left(b_{2} d_{1}+b_{1} d_{2}\right)-a_{2}\left(b_{1} d_{3}-b_{3} d_{1}\right)}{a_{1} b_{2}-a_{2} b_{1}}
\end{aligned}
$$

A partir do sistema na eq. (6), pode-se concluir que:

- O sistema é possível e determinado se $\frac{k_{2}}{k_{1}} \neq 0, \forall k_{1}, k_{2}$, com $k_{1} \neq 0$.

- O sistema é possível e indeterminado se $k_{1}=k_{2}=0$.

- O sistema é impossível se $k_{1}=0$ e $k_{2} \neq 0$.

É importante destacar que, dentro da programação linear, modelos com duas e três variáveis que possuam um sistema de inequações lineares formando as restrições podem ser resolvidos usando o método gráfico. Para tanto, é necessário que o professor faça um trabalho voltado para o lado da geometria analítica, não sendo o caso da proposta deste trabalho. 


\section{Proposta de Ensino}

No intuito de resolver um problema de programação linear usando apenas conteúdos do ensino médio, deve-se primeiramente encontrar os vértices da região delimitada pelas restrições do modelo dado. Estes vértices são equivalentes a um sistema de equações lineares, em que algumas variáveis assumem o valor zero e as demais variáveis têm seus valores determinadas pela resolução do sistema resultante. A partir de todos os vértices encontrados, verifica-se qual deles otimiza a função objetivo do modelo.

Os resultados discutidos adiante foram obtidos a partir de Dantzig (1963), Arenales et al. (2007) e Hillier e Lieberman (2008).

Para um dado modelo de programação linear em sua forma padrão, como o da eq. (1), as soluções que satisfazem simultaneamente as restrições do modelo, ou seja, resolvem o sistema linear, constituem a região de viabilidade do problema. Essa região pode ser visualizada no plano, quando se trabalha com modelos de duas variáveis, ou no espaço, quando se tem três variáveis.

Um ponto da região de viabilidade corresponde a uma solução, chamada viável. Porém, o interesse está na solução viável que otimiza o modelo, chamada de ótima. A partir disto, seja $S$ o conjunto contendo todas as soluções viáveis do modelo, para o qual seguem os resultados adiante.

Resultado 1. O conjunto $S$ é convexo e consiste em um poliedro.

Resultado 2. O conjunto dos pontos extremos (vértices do poliedro) de $S$ é finito e esse número k é tal que $k \leq C_{m}^{n}$, em que $m$ representa o número de equações e $n$ o número de variáveis do sistema linear associado a $S$.

Resultado 3. Seja $x$ um ponto de $S$. Segue que $x$ é uma solução básica viável do modelo se e somente se $x$ for um extremo (vértice do poliedro) de $S$.

Resultado 4. Se no dado modelo de programação linear a função objetivo possuir um máximo (ou um mínimo) finito, então a solução ótima é um ponto extremo de $S$.

Em complemento, podem existir problemas em que a região de viabilidade é vazia ou ilimitada, porém não se tem interesse nestes casos. A proposta é que o professor trabalhe exemplos para os quais se possa aplicador os métodos de resolução de sistemas lineares.
A metodologia usada para a resolução de um modelo de programação linear parte do fato que o problema possui uma solução ótima e o modelo se encontra como a da eq. (1). Caso o modelo não esteja na forma padrão, insere-se em cada restrição uma variável de folga ou de excesso.

$\mathrm{O}$ desenvolvimento adiante considera um exemplo real de uma marcenaria que fabrica dois tipos de cadeiras escolares: uma modelo básica e outra modelo luxo. As cadeiras básicas necessitam de 2 horas para montagem e acabamento, e as cadeiras do modelo luxo necessitam de 3 horas. Para a tapeçaria são necessárias 1 e 2 horas para as cadeiras básicas e luxo, respectivamente. Para a montagem e acabamento das cadeiras existe uma disponibilidade semanal de 260 horas e para a tapeçaria existe uma disponibilidade de 150 horas. Se a receita das cadeiras básicas é de $\mathrm{R} \$ 80$ por unidade e a de cadeiras do modelo luxo é de $\mathrm{R} \$$ 130,00 reais, quantas unidades de cada tipo devem ser produzidas por semana para maximizar a o lucro da marcenaria?

O modelo para o problema da marcenaria envolve duas variáveis e duas restrições com desigualdade do tipo "menor ou igual". São inseridas as variáveis de folga nas restrições no intuito de transformar as desigualdades em igualdades. Após a inserção das variáveis de folga, o modelo passa a ser escrito como:

$$
\begin{aligned}
& \text { Maximizar } z=80 x+130 y \\
& \text { Sujeito a: }\left\{\begin{array}{c}
2 x+3 y+f_{1}=260 \\
x+2 y+f_{2}=150
\end{array}\right.
\end{aligned}
$$

em que $x_{1}, x_{2} \geq 0$ são as variáveis obtidas a partir da modelagem e $f_{1}, f_{2} \geq 0$ são as variáveis de folga. Como há um total de quatro variáveis e duas equações, devem-se combinar as quatro variáveis duas a duas, com um total de $C_{4}^{2}=\frac{4 !}{2 ! 2 !}=6$ possibilidades. Este processo define uma partição do problema, em se define as variáveis básicas e as não básicas a partir de cada combinação.

Segundo Hillier e Lieberman (2008), as variáveis não básicas são definidas a priori atribuindo valor zero, enquanto as básicas são determinadas a partir da resolução do sistema linear resultante. A solução obtida é chamada de solução básica viável caso atenda todas as restrições do modelo original e, partindo do Resultado 3, corresponde a um vértice do poliedro formado a partir do conjunto de restrições. A Tabela 1 apresenta todas as possíveis combinações para o modelo na eq. (9). 
Tabela 1 - Sistemas lineares para o modelo na eq. (9)

\begin{tabular}{|c|c|c|c|}
\hline Variáveis básicas & $\begin{array}{l}\text { Variáveis não } \\
\text { básicas }\end{array}$ & $\begin{array}{l}\text { Sistema linear } \\
\text { resultante }\end{array}$ & $\begin{array}{c}\text { Solução do sistema } \\
\text { linear }\end{array}$ \\
\hline$\left\{\begin{array}{l}x \\
y\end{array}\right.$ & $\left\{\begin{array}{l}f_{1}=0 \\
f_{2}=0\end{array}\right.$ & $\left\{\begin{array}{c}2 x+3 y=260 \\
x+2 y=150\end{array}\right.$ & $\left\{\begin{array}{l}x=70 \\
y=40\end{array}\right.$ \\
\hline$\left\{\begin{array}{l}x \\
f_{1}\end{array}\right.$ & $\left\{\begin{array}{l}y=0 \\
f_{2}=0\end{array}\right.$ & $\left\{\begin{array}{c}2 x+f_{1}=260 \\
x=150\end{array}\right.$ & $\left\{\begin{array}{c}x=150 \\
f_{1}=-40\end{array}\right.$ \\
\hline$\left\{\begin{array}{l}x \\
f_{2}\end{array}\right.$ & $\left\{\begin{array}{l}y=0 \\
f_{1}=0\end{array}\right.$ & $\left\{\begin{array}{c}2 x=260 \\
x+f_{2}=150\end{array}\right.$ & $\left\{\begin{array}{l}x=130 \\
f_{2}=20\end{array}\right.$ \\
\hline$\left\{\begin{array}{l}y \\
f_{1}\end{array}\right.$ & $\left\{\begin{array}{l}x=0 \\
f_{2}=0\end{array}\right.$ & $\left\{\begin{array}{c}3 y+f_{1}=260 \\
2 y=150\end{array}\right.$ & $\left\{\begin{array}{l}y=75 \\
f_{1}=35\end{array}\right.$ \\
\hline$\left\{\begin{array}{l}y \\
f_{2}\end{array}\right.$ & $\left\{\begin{array}{l}x=0 \\
f_{1}=0\end{array}\right.$ & $\left\{\begin{array}{c}3 y=260 \\
2 y+f_{2}=150\end{array}\right.$ & $\left\{\begin{array}{c}y=\frac{260}{3} \\
f_{1}=-\frac{70}{3}\end{array}\right.$ \\
\hline$\left\{\begin{array}{l}f_{1} \\
f_{2}\end{array}\right.$ & $\left\{\begin{array}{l}x=0 \\
y=0\end{array}\right.$ & $\left\{\begin{array}{l}f_{1}=260 \\
f_{2}=150\end{array}\right.$ & $\left\{\begin{array}{l}f_{1}=260 \\
f_{2}=150\end{array}\right.$ \\
\hline
\end{tabular}

Note que a solução do sistema $\left\{\begin{array}{c}2 x+f_{1}=260 \\ x=150\end{array}\right.$, conforme a Tabela 1 , resulta em $x=150$ e $f_{1}=-40$. Como as variáveis só podem assumir valores reais não negativos, tal solução não pertence a região viável do problema. O mesmo ocorre para a solução $(x, y)=$ $\left(0, \frac{260}{3}\right)$.

Em seguida, o professor deve solicitar aos alunos para que calculem o valor da função objetivo, conforme se tem na Tabela 2, dada a solução de cada sistema linear da Tabela 1. Os alunos devem analisar, dentre os valores objetivo obtidos, para qual o máximo ocorre. Neste caso, chega-se na solução $x=70, y=40, f_{1}=0$ e $f_{2}=0$. Em outras palavras, a marcenaria deve produzir 70 cadeiras do tipo básico e 40 cadeiras do tipo luxo, resultando em lucro máximo equivalente a 10.800 reais.

Tabela 2 - Valor da função objetivo para as soluções na Tabela 1

\begin{tabular}{|c|c|c|}
\hline Sistema linear resultante & Solução do sistema & Valor da função $z=F(x, y)$ \\
\hline$\left\{\begin{array}{c}2 x+3 y=260 \\
x+2 y=150\end{array}\right.$ & $\left\{\begin{array}{l}x=70 \\
y=40\end{array}\right.$ & $\mathrm{F}(70,40)=10.800$ \\
\hline$\left\{\begin{array}{c}2 x+f_{1}=260 \\
x=150\end{array}\right.$ & $\left\{\begin{array}{l}x=150 \\
f_{1}=-40\end{array}\right.$ & Ponto inviável \\
\hline$\left\{\begin{array}{c}2 x=260 \\
x+f_{2}=150\end{array}\right.$ & $\left\{\begin{array}{l}x=130 \\
f_{2}=20\end{array}\right.$ & $\mathrm{F}(130,0)=10.400$ \\
\hline$\left\{\begin{array}{c}3 y+f_{1}=260 \\
2 y=150\end{array}\right.$ & $\left\{\begin{array}{l}y=75 \\
f_{1}=35\end{array}\right.$ & $\mathrm{F}(0,75)=9.750$ \\
\hline$\left\{\begin{array}{c}3 y=260 \\
2 y+f_{2}=150\end{array}\right.$ & $\left\{\begin{array}{l}y=\frac{260}{3} \\
f_{1}=-\frac{70}{3}\end{array}\right.$ & Ponto inviável \\
\hline$\left\{\begin{array}{l}f_{1}=260 \\
f_{2}=150\end{array}\right.$ & $\left\{\begin{array}{l}f_{1}=260 \\
f_{2}=150\end{array}\right.$ & $\mathrm{F}(0,0)=0$ \\
\hline
\end{tabular}


Os sistemas lineares descritos na Tabela 2 identificam cada um dos vértices da região viável, ou seja, as soluções determinam todas as possibilidades a candidatos de vértices da região. Note que o método proposto exclui soluções que não pertencem à região viável ao observar o domínio das variáveis.

Em resumo, a metodologia para trabalhar com programação linear na perspectiva da resolução de sistemas de equações lineares é simples e objetiva. $\mathrm{O}$ estudante não terá dificuldades com a abordagem, pois a mesma não utiliza e nem apresenta novos conceitos, além daqueles já estudados durante o ensino médio. Ao contrário, a metodologia permite que os alunos enxerguem situações problema do cotidiano dentro da sala de aula, bem como permite resolvê-las de forma eficiente.

É importante ressaltar que as etapas anteriores para resolver o modelo consistem em passos básicos dentro da teoria da programação linear. Além disso, quando o modelo cresce com relação ao número de variáveis e equações, esta metodologia se torna extremamente ineficiente na prática, pois requer um grande número de operações. Daí, o uso de métodos mais eficientes, como o Simplex, detalhado em Dantzig (1963), porém que requerem um maior aprofundamento no tema, muitas vezes impossível de ser feito durante o ensino médio pela não flexibilização do currículo escolar.

\section{Uso de um Software Matemático}

Um passo importante no processo de ensino considera a inclusão de softwares como possibilidade de construção do conhecimento (GOMES, 2001). No caso da proposta em questão, que usa sistemas lineares, a resolução de sistemas com $m$ equações e $n \geq 3$ variáveis é oneroso, em especial, pelo fato da quantidade de sistemas crescer da forma $C_{m}^{n}$.

A partir disto, a ideia é combinar a resolução dos sistemas resultantes por meio de um software matemático livre, o Octave ${ }^{\circledR}$. Ao determinar os sistemas lineares resultantes, a solução de cada um é obtida usando o respectivo software.

Para se trabalhar com o Octave, o professor precisa inicialmente introduzir a ideia do software, sua história e objetivos, e alguns comandos básicos, especialmente aqueles voltados para a resolução de sistemas de equações lineares (EATON; BATEMAN; HAUBERG, 2008). Considera-se um sistema genérico, de ordem $3 \times 3$, que pode ser escrito como $\mathrm{AC}=\mathrm{B}$, com:

$A=\left(\begin{array}{lll}a_{1} & b_{1} & c_{1} \\ a_{2} & b_{2} & c_{2} \\ a_{3} & b_{3} & c_{3}\end{array}\right), B=\left(\begin{array}{l}d_{1} \\ d_{2} \\ d_{3}\end{array}\right)$ e $C=\left(\begin{array}{l}x \\ y \\ Z\end{array}\right)$

Se a matriz $A$ for invertível, então a solução é encontrada fazendo $C=A^{-1} B$. Para um modelo com $m=3$ equações e $n=6$ variáveis, sendo três, variáveis de decisão e três de folga, é necessário combinar as seis variáveis três a três, totalizando $C_{6}^{3}=\frac{6 !}{3 ! 3 !}=20$. Seguindo a proposta, deve-se escolher $(n-m)=3$ variáveis para serem as não básicas, cujo valor é zero, enquanto as demais são determinadas pela resolução do sistema resultante. Considere o modelo na eq. (11) tomado como exemplo para ilustrar o uso do software.

Maximizar $z=40 x_{1}+100 x_{2}+120 x_{3}$

Sujeito a:

$$
\left\{\begin{array}{c}
4 x_{1}+1,25 x_{2}+x_{3}+f_{1}=200 \\
20 x_{1}+50 x_{2}+60 x_{3}+f_{2}=6000 \\
5 x_{1}+15 x_{2}+25 x_{3}+f_{3}=3000 \\
x_{1} \geq 0, x_{2} \geq 0, x_{3} \geq 0 \\
f_{1} \geq 0, f_{2} \geq 0, f_{3} \geq 0
\end{array}\right.
$$

sendo $f_{1}, f_{2}$ e $f_{3}$ as variáveis de folga, acrescentadas ao modelo para se atingir a igualdade nas restrições. Dado que há um total de 20 possíveis sistemas, o sistema para o qual $x_{1}, x_{2}$ e $f_{2}$ são variáveis não básicas é:

$$
\left\{\begin{array}{c}
x_{3}+f_{1}=200 \\
60 x_{3}=6000 \\
25 x_{3}+f_{3}=3000
\end{array}\right.
$$

O professor precisa primeiro mostrar aos alunos como montar as matrizes $A$ e $B$ seguindo a eq. (10). Em seguida, inserir cada uma delas usando a linha de comando do Octave. A Figura 1 ilustra o processo, sendo a matriz $A$ inserida primeiro e, em seguida, a matriz $B$. 


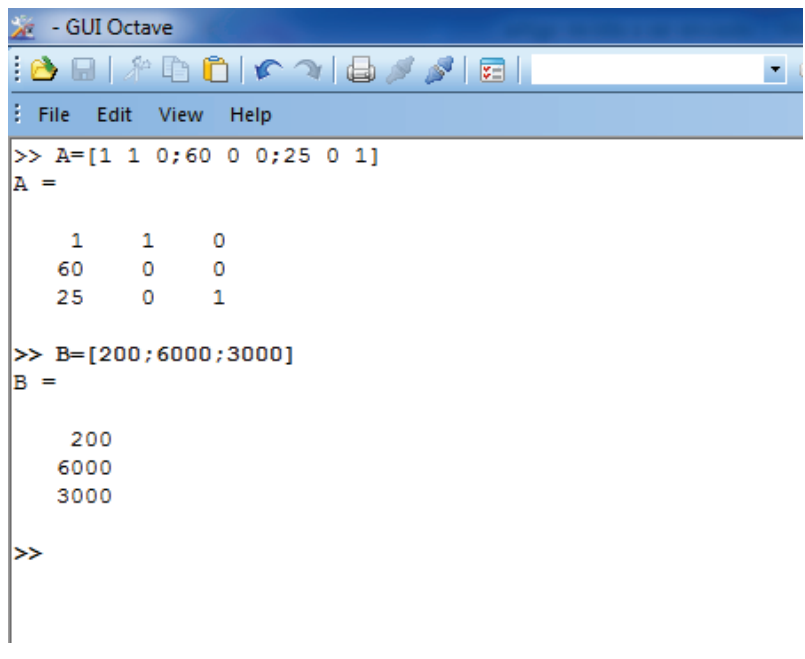

Figura 1 - Inserção das matrizes $A$ e $B$ pela linha de comando

A solução do sistema $A C=B$ segue naturalmente por meio do comando $C=A \backslash B$. Note que foi encontrada a solução $x_{3}=100, f_{1}=$ 100 e $f_{3}=500$, conforme apresentado na Figura 2 .

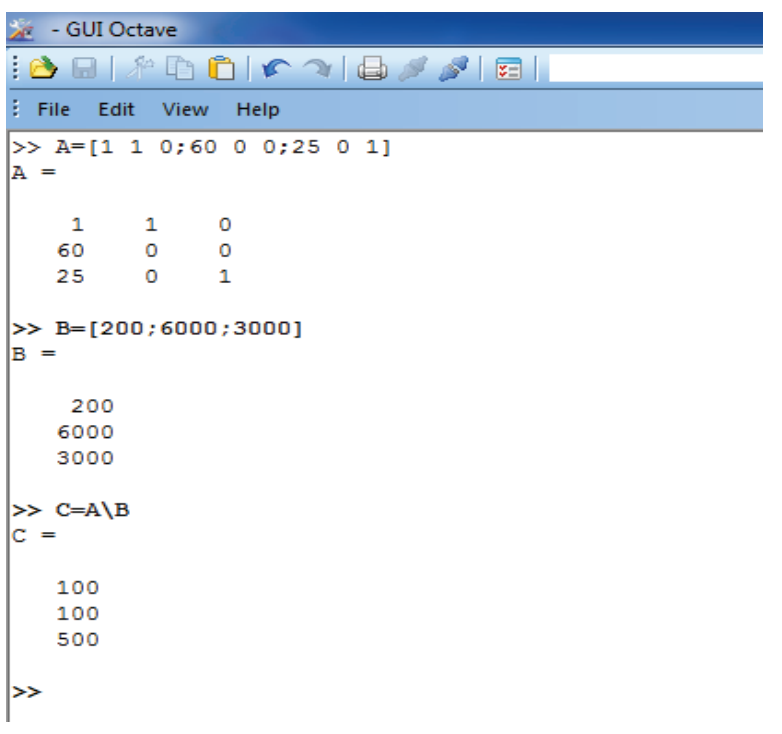

Figura 2 - Resolução do sistema linear na eq. (12)

Após encontrado o valor das variáveis, determina-se o valor da função objetivo. $\mathrm{O}$ comando utilizado é $\mathrm{z}=120^{*} \mathrm{C}(3,1)$, uma vez que $x_{1}$ e $x_{2}$ são nulas e $f_{1}$ e $f_{3}$ não entra na função objetivo. A Figura 3 apresenta o uso do comando, tal que a solução obtida corresponde a $z=12.000$.

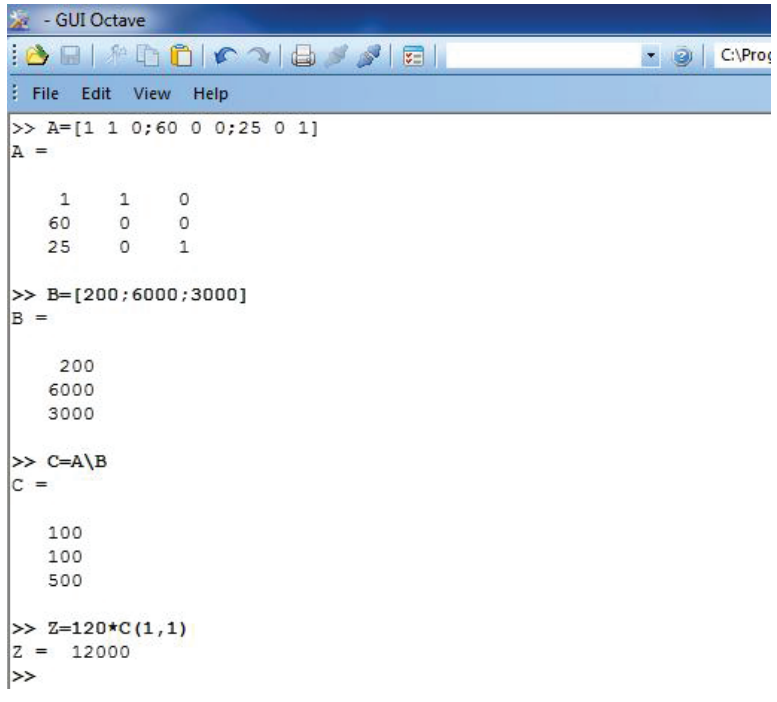

Figura 3 -. Valor da função objetivo dada a solução do sistema na eq. (12)

A estratégia apresentada para resolver o sistema linear na eq. (12) é, então, aplicada para resolver os 20 sistemas lineares do modelo na eq. (11). O professor deve frisar que os mesmos passos podem ser aplicados para resolver um sistema qualquer, com $m$ equações e $n$ variáveis, e mostrar que o software atua como uma ferramenta facilitadora para os cálculos. Porém, o professor precisa alertar que o uso do software só é interessante quando se conhece a teoria por trás do método utilizado, pois na ocorrência de erros, o aluno terá pré-requisito para análise e, dentro das possibilidades, realizar a correção do erro.

\section{Conclusões e Trabalhos Futuros}

Este trabalho busca pela inserção da Programação Linear no ensino médio como uma aplicação natural de sistemas de equações lineares. Partindo de problemas simples, com aplicações reais que podem ser contextualizados, é que os alunos do ensino médio têm um primeiro contato com essa técnica poderosa, a programação linear. Madeira (1998) defendeu em seu trabalho o ensino por meio de uma abordagem contextualizada na prática.

Apesar da proposta aqui apresentada não ter sido aplicada em sala de aula, pode-se concluir que a proposta permite trabalhar a matemática de uma forma contextualizada e com situações problemas do dia a dia dos alunos. De forma similar, os autores Melo (2012), Almeida (2013) e 
Silva (2013) também chegaram em tal conclusão ao trabalhar com a Programação Linear no contexto do ensino médio.

Como o estudo de sistemas lineares está presente no currículo atual conjuntamente com a utilização da modelagem matemática como metodologia de ensino, essa proposta tem ainda a vantagem de ser aplicada em ambientes em que não há a flexibilização do currículo para a inserção de novos conteúdos. No entanto, quando existe a possibilidade de flexibilização do currículo, torna-se interessante o professor fazer uso do método Simplex.

Para lidar com problemas maiores, o professor pode contar também com o auxílio de um software matemático, em que os cálculos passam a ser feitos com rapidez. Outra vantagem de se combinar o ensino por meio do software está na possibilidade de trabalhar com a resolução gráfica em paralelo a resolução dos sistemas lineares.

Como proposta para trabalhos futuros, destaca-se uma nova proposta para integrar o ensino do método simplex no ensino médio. A ideia é dividir o método em partes e tratar cada uma dessas nos conteúdos que são obrigatórios no currículo, como: sistemas de equações lineares, geometria analítica e combinatória. Ao final, o professor sistematiza cada uma das etapas que foram estudadas para, então, apresentar o método Simplex. Por outro lado, uma extensão natural deste trabalho consiste em aplica-lo dentro da sala de aula e a partir do feedback dos alunos, o professor adequa os conteúdos a serem trabalhados.

\section{Agradecimentos}

Os autores agradecem o apoio financeiro recebido das agências: CAPES, CNPq e FAPEG.

\section{Referências}

ALMEIDA, R. C. O teorema fundamental da programação linear e modelagem matemática no ensino médio. 2011. 130 f. Dissertação (Mestrado Profissional em Matemática), Universidade Federal de São João Del Rei - UFSJ. São João Del Rei, 2011.
ARENALES, M. N.; ARMETANO. V.; MORABITO, R.; YANASSE, H. Pesquisa Operacional. Rio de Janeiro: Campus, 2007.

BASSANEZI, R. C. Ensino-aprendizagem com modelagem matemática. São Paulo: Contexto, 2002.

BIEMBENGUT, M. S.; Hain, N. Modelagem matemática no ensino. São Paulo: Contexto, 2000.

BOLDRINI, J. L.; COSTA, S. I. R.; FIGUEIREDO, V. L.; WETZLER, H. G. Álgebra Linear. 3a ed. São Paulo: Harbra, 1980.

DANTZIG, G. B. Linear programming and extensions. Princeton: Princeton University Press and RAND Corporation, 1963.

FIORENTINI, D. Alguns modos de ver e conceber o ensino da matemática no Brasil. Zetetiké, 3(4): 1-37, 1995.

GOMES, C. Em busca de um modelo psico-educativo para a avaliação de softwares educacionais. 2001. 138 f. Dissertação (Programa de Pós Graduação em Engenharia de Produção), Universidade Federal de Santa Catarina - UFSC, Florianópolis, 2001.

HILLIER, F. S.; LIEBERMAN, G. J. Introdução à Pesquisa Operacional. 9a ed. Porto Alegre: AMGH, 2008.

HOWARD, A.; RORRES, C. Álgebra Linear com Aplicações. 8a ed. Porto Alegre: Bookman, 2001.

LEON, S. J. Álgebra Linear com Aplicações. 8a ed. Rio de Janeiro: LTC, 2011.

MADEIRA, M. C. (org.). Representações sociais e educação: algumas reflexões. Natal: EDUFRN, 1998.

MELO, J. N. B. Uma Proposta de Ensino Médio e Aprendizagem de Programação Linear no Ensino Médio. 2012. 124 f. Dissertação (Mestrado em Ensino da Matemática) - Universidade Federal do Rio Grande do Sul - UFRGS, Porto Alegre, 2012.

POLYA, G. A arte de resolver problemas: Um novo aspecto do método matemático. Rio de Janeiro: Interciência, 1995. 
SILVA, K. Modelagem Matemática com Programação Linear: Uma proposta de trabalho no Ensino Médio. 2013. 107 f. Dissertação (Mestrado Profissional em Matemática), Universidade Estadual do Sudoeste da Bahia - UESB. Vitória da Conquista, 2013.

EATON, J. W.; BATEMAN, D.; HAUBERG. S. GNU Octave. 3a ed. Network Theory Limited, 2008.

ZEICHNER, K. A formação reflexiva de professores: Ideias e práticas. Lisboa: Educa, 1993. 